\title{
Development of a relativistic coupled-cluster method for one electron detachment theory: Application to Mn IX, Fe X, Co XI and Ni XII ions
}

\author{
D. K. Nandy ${ }^{*}$ and B. K. Sahoo ${ }^{\dagger}$ \\ Theoretical Physics Division, Physical Research Laboratory, Ahmedabad-380009, India
}

(Dated: Received date; Accepted date)

\begin{abstract}
We have developed one electron detachment theory from a closed-shell atomic configuration in the relativistic Fock-space coupled-cluster ansatz. Using this method, we determine sensitivity coefficients to the variation of the fine structure constant in the first three important low-lying transitions of the astrophysically interesting highly charged Mn IX, Fe X, Co XI and Ni XII ions. The potential of this method has been assessed by evaluating the detachment energies of the removed electrons and determining lifetimes of the atomic states in the above ions. To account the sensitivity of the higher order relativistic effects, we have used the four component wave functions of the DiracCoulomb-Breit Hamiltonian with the leading order quantum electrodynamics (QED) corrections. A systematic study has been carried out to highlight the importance of the Breit and QED interactions in the considered properties of the above ions.
\end{abstract}

PACS numbers: 31.10.+z, 31.15.A-, 31.15.ag, 31.15.ap

\section{INTRODUCTION}

New field of research in the investigation of temporal variation of the fundamental constants has been gaining the ground steadily in both the theoretical and experimental physics for the past few years [1-4]. The important aspects for searching this variation are to establish theories suggesting violation of the Einstein's equivalence principle and to support the models like Kaluza-Klein theory that attempts to unify gravity with the other three unified fundamental interactions [5 11]. This may also probe the multi-dimensionality to space as predicted by the superstring theories [12]. These theories predict temporal variation of the fundamental constants including the electromagnetic fine structure constant $\left(\alpha_{e}=\frac{e^{2}}{\hbar c}\right)$ in the low energy limit at the cosmological time-scale.

From the experimental front, the signature of possible variation of $\alpha_{e}$ can be observed from three classes of measurements. Geophysical method: The data obtained from the isotopic decay in the natural radioactive reactor at the Oklo observatory can be used as a tool to probe the variation of $\alpha_{e}$ as these isotopes corresponds to typically $10^{9}$ years old and signature of the discrepancy between the decay rate with the present laboratory value indicates a small deviation in the $\alpha_{e}$ value in this time scale [1]. Atomic clock method: Also, the high precision frequency measurements using the atomic and the singly charged ionic clocks can be used to probe variation of $\alpha_{e}$ in an elegant manner. In these experiments the time dependency of $\alpha_{e}$ is inferred by comparing the transition frequencies between at least two clocks [1, 2, 13, 14]. The main advantage of these types of experiments is related to their efficient control over the systematic errors. However, the time period involved to carry out these measurements is

\footnotetext{
*Email: dillip@prl.res.in

${ }^{\dagger}$ Email: bijaya@prl.res.in
}

of the order of few years only and the typical choice of the candidates for the clocks are either the neutral atoms or the singly charged ions where the relativistic enhancements are typically small. Astrophysical method: The most natural way of finding out the evidence of possible variation of this constant is by analyzing the atomic and molecular absorption spectra coming out of distant astronomical objects such as the high-red-shifted quasars [15 18]. The time scale at which these events are occurred corresponds to again $10^{7}-10^{9}$ years back and the statistical uncertainties in these systems can be reduced using the many-multiplet methods [19, 20]. The impressive part of considering astrophysical investigation of variation of $\alpha_{e}$ is that one can consider a large number of spectral lines for the analysis. As a matter of fact, the spectral lines from the highly charged ions can be investigated in this case which can have extra ordinary large enhancement of the relativistic effects 21, 22]. In this paper, we analyze the relativistic sensitivity coefficients in Mn IX, Fe X, Co XI and Ni XII ions which are not explored before.

The considered Mn IX, Fe X, Co XI and Ni XII ions are of particular interest for analyzing their spectra as the ground states of these ions have the fine structure splittings. The transitions among these states can occur through the forbidden transitions and their wavelengths lie in the ultra-violet (UV) region [23]. The next excited levels are the $s$-states which can decay to the above two lower states through the allowed channel. For an advantage, these transitions have wavelengths in the optical region [23]. It can be noticed that the fine structure transitions have one more leading order relativistic correction in $\alpha_{e}^{2}$ than the optical transitions. As a fact, the ratios of transition frequencies in the above ions seem to be very promising quantities for carrying out the investigation of any temporal variation of $\alpha_{e}$ by comparing these values from the spectra coming out of any astronomical objects with their corresponding laboratory values. It is identified from the solar extreme ultraviolet (EUV) spectra 
line that most of them are the emission lines of Fe $\mathrm{X}$ ion [24 29]. These lines can be used to extract data for the electron density in the solar corona as proposed by Jordan [30]. Mason and Nussbaumer had also observed that under typical solar coronal condition, $\mathrm{Cl}$ like ions such as $\mathrm{Fe} \mathrm{X}$ give rise to most of the prominent spectra 31]. The red iron line corresponds to the forbidden transition between the fine structure levels of the ground state of Fe X. The other ions Co XI and Ni XII are also important for the astrophysical study, but they are relatively less abundant in the astrophysical objects. Quite a few emission lines of Co XI are observed in the solar plasma, and in the spectra from theta-pinch plasma $[26,32-34]$. Some of the lines of Co XI ranging the wavelengths in between 65$340 \AA$ have been observed and tabulated in 35 . It is also revealed from the data analysis of high-resolution soft X-ray spectrum of nearby F-type star Procyon that the emission lines contains spectra from Mn IX and Ni XII along with from other highly charged ions [36]. Moreover, these ions can be reproduced using the accelerators for their laboratory studies. Thus, theoretical calculations of the spectral properties in these ions are necessary.

Theoretical determination of the atomic states in the considered ions are very challenging owing to the fact that they contain five valence electrons in their outer most orbitals. There have been only few studies carried out for the evaluation of the lifetimes of the first excited states using semi-empirical, mean-field and configuration interaction (CI) methods; however there are neither any theoretical calculations nor any observations of the lifetimes of the second excited states are available in the considered ions. In fact, the higher order relativistic effects are never investigated in these ions. We have developed here an all order perturbative method in the relativistic coupled-cluster (RCC) framework to carry out the study of correlation effects and relativistic corrections systematically in the first three low-lying states of the undertaken ions and would like to calculate precisely the electron detachment energies, the sensitivity coefficients for the variation of $\alpha_{e}$ involving the first three transitions and the lifetimes of the first two excited states. We shall also demonstrate the roles of the higher order relativistic effects in the estimation of the sensitivity coefficients.

\section{THEORY AND METHOD OF CALCULATIONS}

\section{A. $\alpha_{e}$ Sensitivity Coefficient}

The energy expression for a state of any multi-electron atomic system can be approximated to [37]

$$
E_{n} \simeq c^{2}\left(Z \alpha_{e}\right)^{2}\left\{\frac{1}{2 n^{2}}+\frac{\left(Z \alpha_{e}\right)^{2}}{2 n^{3}}\left(\frac{1}{|\kappa|}-\frac{3}{4 n}\right)\right\}
$$

where $Z$ is the atomic number of the atom, $c$ is the velocity of light, $n$ is the principal quantum number of the state and $\kappa= \pm\left(j+\frac{1}{2}\right)$ is the relativistic quantum number with angular momentum of the state $j$. Since the relativistic effects to the energy levels close to the nucleus are large due to the high angular velocity of the electron, the relativistic corrections to the energy levels can be approximated to [38]

$$
\Delta=-\frac{Z_{a}^{2}}{2} \frac{\left(Z \alpha_{e}\right)^{2}}{\nu^{3}}\left(\frac{1}{j+1 / 2}-\frac{Z_{a}}{Z \nu}\left[1-\frac{Z_{a}}{4 Z}\right]\right),
$$

with $\nu$ is the effective principal quantum number and $Z_{a}$ is the effective charge seen by an electron after the screening effect of the inner core electrons. As the atomic energy levels scale of the order of $\alpha_{e}^{2}$ after taking the relativistic correlation effects into account, hence the transition frequencies among the atomic levels are very sensitive to a small change in $\alpha_{e}$ value which will, obviously, get enhanced for a large atomic number $Z$ and for a small value of $\nu$. Therefore, this sensitivity is large in the highly charged ions. For the theoretical investigation, this sensitivity can be estimated by considering a relativistic method to calculate transition frequency $(\omega)$ of a transition by expressing them into

$$
\omega(x) \approx \omega_{0}+q x
$$

where $\omega_{0}$ corresponds to transition frequency with the laboratory value of the fine structure constant $\alpha_{0}, x=$ $\left(\frac{\alpha_{e}}{\alpha_{0}}\right)^{2}-1$ is the Taylor coefficient of the first derivative of $\omega$ and $q=\left.\frac{d \omega}{d x}\right|_{x=0}$ is known as the sensitivity coefficient for the variation of the fine structure constant. For the numerical estimate of the $q$-factor, it can be evaluated at the first order correction in $\alpha_{e}^{2}$ by

$$
q \approx \frac{\omega(+x)-\omega(-x)}{2 x},
$$

for a given choice of small value of $x$ which, in the present calculation, is chosen as 0.05 .

\section{B. Lifetime of an Atomic State}

The transition probabilities due to the E1, M1 and E2 channels of an atomic transition $\left|\Psi_{f}\right\rangle \rightarrow\left|\Psi_{i}\right\rangle$ are given by [39]

$$
\begin{aligned}
A_{f i}^{E 1} & =\frac{2.0261 \times 10^{-6}}{\lambda_{f i}^{3} g_{f}} S_{f i}^{E 1}, \\
A_{f i}^{E 2} & =\frac{1.1195 \times 10^{-22}}{\lambda_{f i}^{5} g_{f}} S_{f i}^{E 2}
\end{aligned}
$$

and

$$
A_{f i}^{M 1}=\frac{2.6971 \times 10^{-11}}{\lambda_{f i}^{3} g_{f}} S_{f i}^{M 1},
$$

where the quantity $S_{f i}^{O}=\left|\left\langle\Psi_{f}|| O \| \Psi_{i}\right\rangle\right|^{2}$ is known as the line strength of a transition for a corresponding channel $O$ and is estimated here in atomic unit (au), the transition 


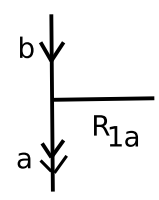

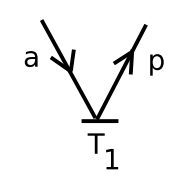
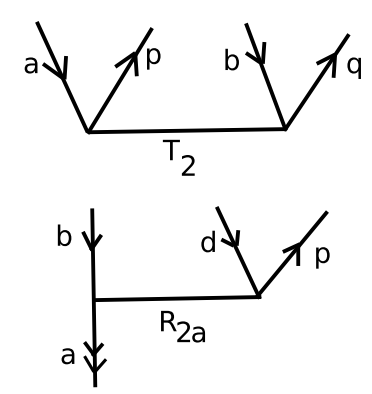

FIG. 1: Goldstone diagrammatic representation of the $T$ and $R_{a}$ operators. Horizontal lines represent the reference state $\left|\Phi_{0}\right\rangle$, line with index $p$ going up from the reference means creation of an electron in the virtual $p$ orbital (similarly line $p$ coming into the reference line from below means electron annihilation from a virtual orbital $p$ as used in the latter figures), line with index $a$ going down from the reference means annihilation of the electron from the occupied orbital $a$ and $b$ line coming to the reference means it is creating an electron in the occupied orbital $b$.

wavelength $\lambda_{f i}$ is taken in $\mathrm{cm}$ and $g_{f}=2 J_{f}+1$ is the degeneracy factor with the angular momentum $J_{f}$ of the state $\left|\Psi_{f}\right\rangle$. The determined transition probabilities are obtained in $s^{-1}$ from the above quantities.

The emission (absorption) oscillator strengths $f_{f i}\left(f_{i f}\right)$ due to the above transition probabilities are given by [40]

$$
f_{f i}=1.4992 \times 10^{-24} A_{f i} \frac{g_{f}}{g_{i}} \lambda_{f i}^{2}
$$

which follows that $g_{i} f_{i f}=-g_{f} f_{f i}$.

The reduced matrix elements for the single particle orbitals corresponding to E1, M1 and E2 transitions are given by [39]

$$
\begin{aligned}
\left\langle\kappa_{f}\|e 1\| \kappa_{i}\right\rangle= & \left\langle\kappa_{f}\left\|C^{(1)}\right\| \kappa_{i}\right\rangle \\
& \int_{0}^{\infty} d r r\left(P_{f}(r) P_{i}(r)+Q_{f}(r) Q_{i}(r)\right),
\end{aligned}
$$

$$
\begin{aligned}
\left\langle\kappa_{f}\|m 1\| \kappa_{i}\right\rangle= & \frac{\left(\kappa_{f}+\kappa_{i}\right)}{\alpha}\left\langle-\kappa_{f}\left\|C^{(1)}\right\| \kappa_{i}\right\rangle \\
& \int_{0}^{\infty} d r r\left(P_{f}(r) Q_{i}(r)+Q_{f}(r) P_{i}(r)\right),
\end{aligned}
$$

and

$$
\begin{aligned}
\left\langle\kappa_{f}\|e 2\| \kappa_{i}\right\rangle= & \left\langle\kappa_{f}\left\|C^{(2)}\right\| \kappa_{i}\right\rangle \\
& \int_{0}^{\infty} d r r^{2}\left(P_{f}(r) P_{i}(r)+Q_{f}(r) Q_{i}(r)\right),
\end{aligned}
$$

where $P(r)$ and $Q(r)$ denote the large and small components of the radial parts of the single particle Dirac orbitals, respectively. The reduced Racah coefficients are

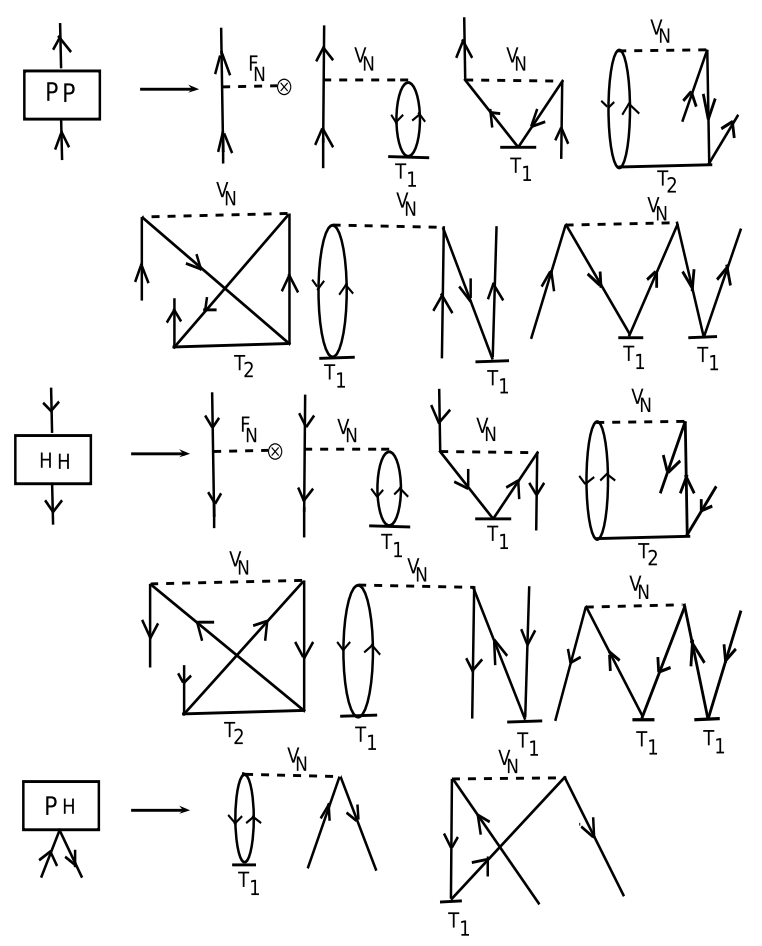

FIG. 2: Effective one-body diagrams constructed from $\widehat{H_{N} e^{T}}$ for the evaluation of the electron detachment energy and the $R_{a}$ amplitudes.

given by

$$
\begin{aligned}
\left\langle\kappa_{f}\left\|C^{(k)}\right\| \kappa_{i}\right\rangle= & (-1)^{j_{f}+1 / 2} \sqrt{\left(2 j_{f}+1\right)\left(2 j_{i}+1\right)} \\
& \left(\begin{array}{ccc}
j_{f} & k & j_{i} \\
1 / 2 & 0 & -1 / 2
\end{array}\right) \pi\left(l_{\kappa_{f}}, k, l_{\kappa_{i}}\right),
\end{aligned}
$$

with

$$
\pi\left(l, k, l^{\prime}\right)= \begin{cases}1 & \text { for } l+k+l^{\prime}=\text { even } \\ 0 & \text { otherwise }\end{cases}
$$

The lifetime of a given atomic state is the inverse of the total transition probabilities involving all possible spon(10) taneous emission channels. i.e. the lifetime (in $s$ corresponding to the aforementioned units) of the state $\left|\Psi_{k}\right\rangle$ is given by

$$
\tau_{k}=\frac{1}{\sum_{O, i} A_{k i}^{O}}
$$

where the sum over $O$ represents all possible decay channels due to the transition operators $O$ and the sum over $i$ corresponds to all the lower transition states. 


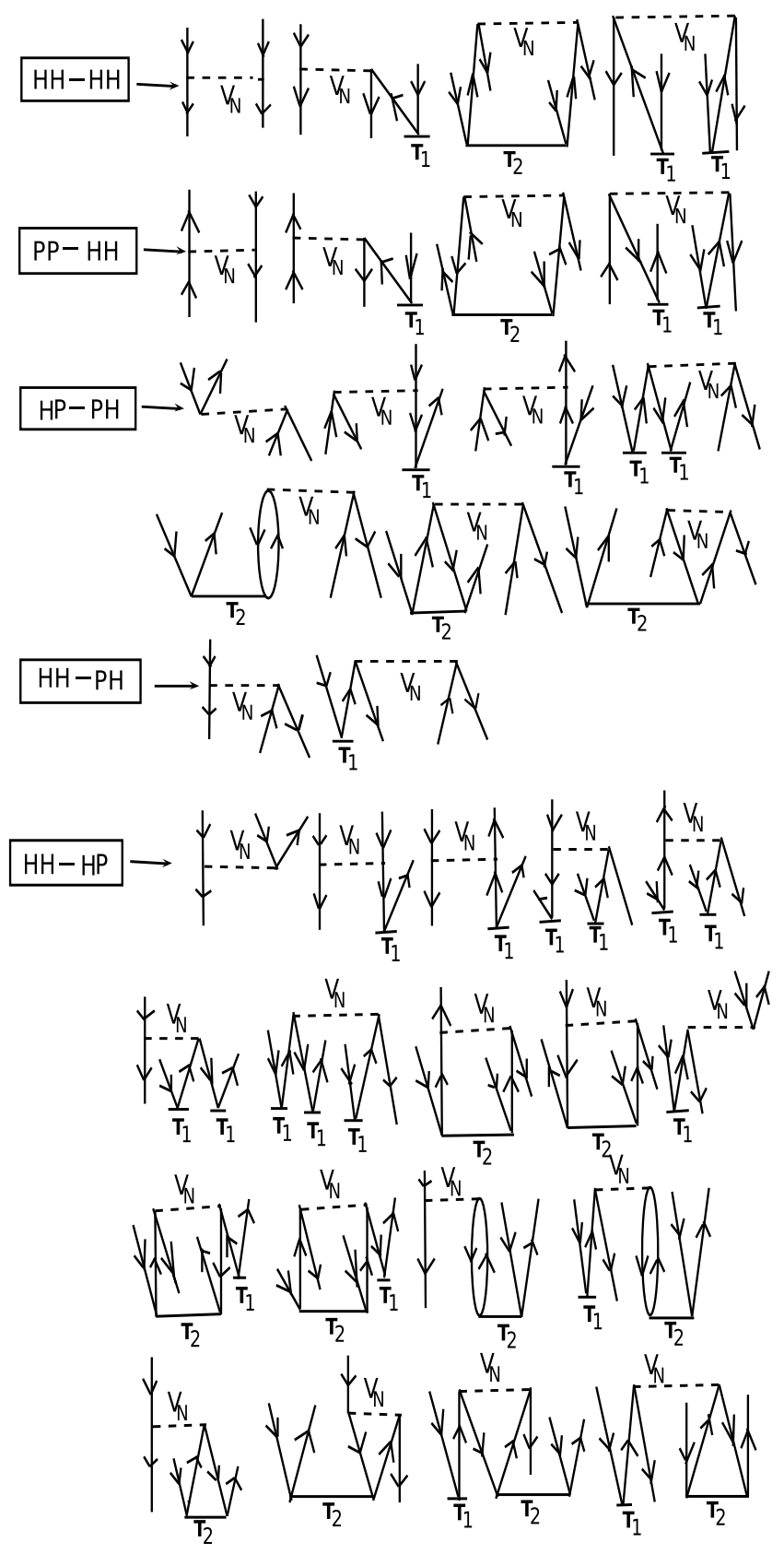

FIG. 3: Effective two-body diagrams constructed from $\widehat{H_{N} e^{T}}$ for the calculation of the electron detachment energy and the $R_{a}$ amplitudes.

\section{RCC method for electron detachment}

The ground and the first two excited states, those are of particular interest in the present work, of the considered ions have a structure of one electron less than the closed-shell configuration $\left[3 s^{2} 3 p^{6}\right]$. These states can be generated by removing one electron from the respective $3 p_{3 / 2}, 3 p_{1 / 2}$ and $3 s$ orbitals in three separate steps. Construction of the atomic state function (ASF) allowing couplings between all possible configuration state func-

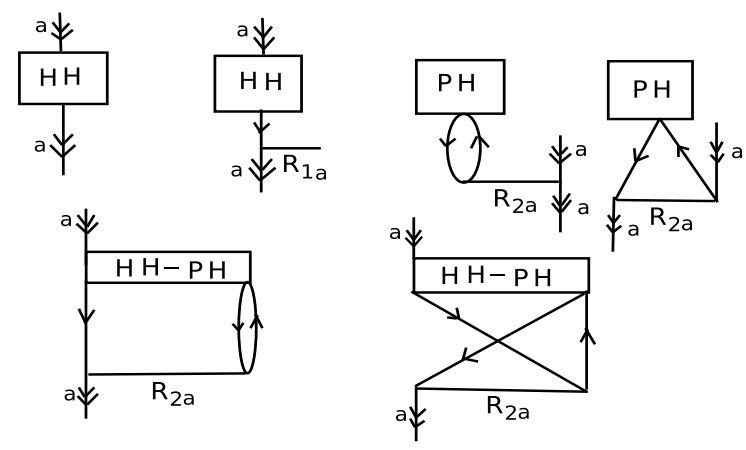

FIG. 4: Diagrams contributing to the calculations of the detachment energies. The line with double arrow means the orbital $a$ from which an electron has been removed.

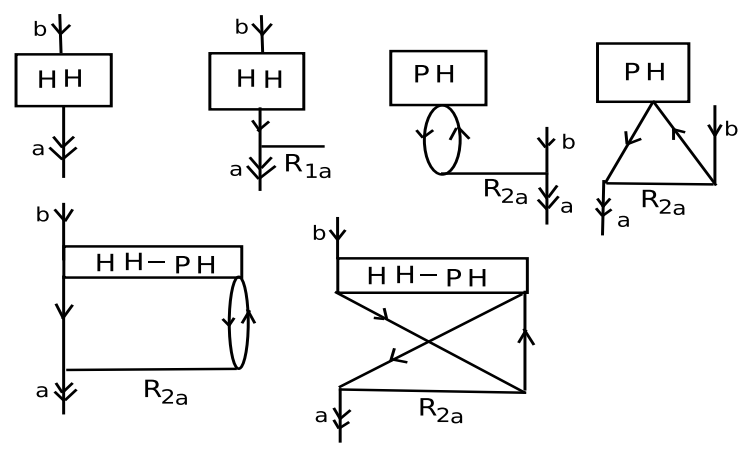

FIG. 5: Diagrams for the evaluation of the $R_{1 a}$ amplitudes. The first diagram arises from the right hand side (rhs) and the rest are from the left hand side (lhs) of Eq. (29).

tions (CSFs) having the same angular momentum is not easily viable in these ions owing to the presence of many electrons in the valence space. One of the approachable ways of calculating these ASFs is to evaluate wave function for the $\left[\begin{array}{ll}3 s^{2} & 3 p^{6}\end{array}\right]$ configuration by accounting correlations among all these electrons and later remove an electron from the respective orbital in a Fock-space representation. In this procedure, one has the flexibility to use the reduced matrix elements for minimizing the computational requirements so that it can afford to include the correlation effects more efficiently.

The other important aspect is to construct a perturbative method for the inclusion of the correlation effects among the electrons to infinite order for the high precision calculations of the wave functions. Keeping in mind the above mentioned complexity in the mixing of CSFs in order to obtain the desired ASFs in the considered ions, the developed (Z-1) Fock-space based coupled-cluster method in this work in the relativistic frame work (here onwards we refer it to RCC method) seems to be one of the most elegant approaches to employ in a closed-core with $Z$ 

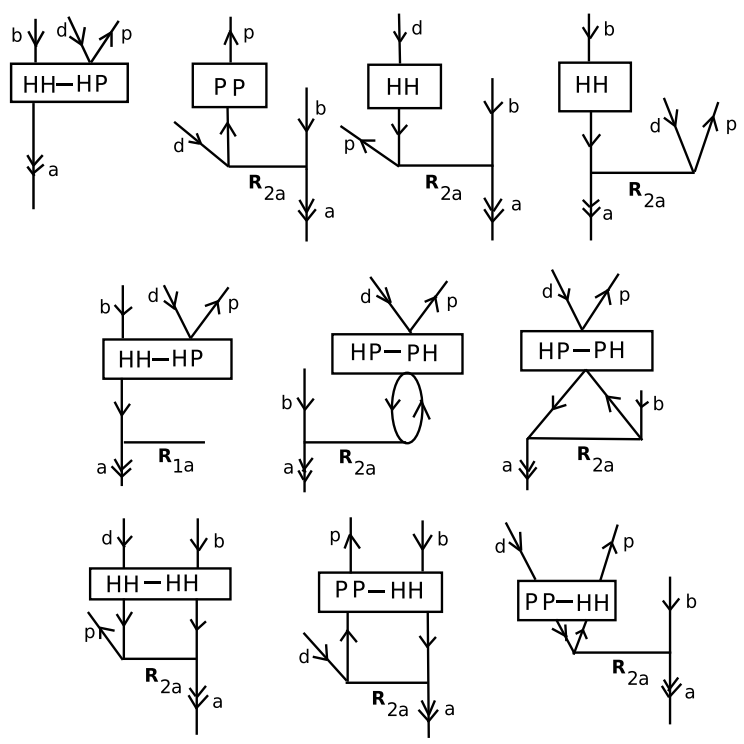

FIG. 6: Diagrams for the evaluation of the $R_{2 a}$ amplitudes. The first diagram arises from the right hand side (rhs) and the rest are from the left hand side (lhs) of Eq. (30).

number of electrons and generate the atomic states of its ion by removing one electron. To describe this procedure, we express the above ASFs in the form

$$
\left|\Psi_{a}\right\rangle=a_{a}\left|\Psi_{0}\right\rangle+R_{a} a_{a}\left|\Psi_{0}\right\rangle,
$$

where $\left|\Psi_{0}\right\rangle$ represents ASF of the $\left[3 s^{2} 3 p^{6}\right]$ configuration and $a_{a}$ represents annihilation of the electron from the $a$ orbital of the closed-core. The important point to be noticed here is that we have already accounted correlations among all the electrons from the $\left[3 s^{2} 3 p^{6}\right]$ configuration in the construction of $\left|\Psi_{0}\right\rangle$. The $R_{a}$ operator, thus, need to remove the extra correlation effects that is being taken into account for the extra $a_{a}$ electron in the determination of $\left|\Psi_{a}\right\rangle$. In the RCC ansatz, the above expression yields to

$$
\begin{aligned}
\left|\Psi_{a}\right\rangle & =a_{a} e^{T}\left|\Phi_{0}\right\rangle+R_{a} a_{a} e^{T}\left|\Phi_{0}\right\rangle \\
& =e^{T}\left(1+R_{a}\right) a_{a}\left|\Phi_{0}\right\rangle \\
& =e^{T}\left(1+R_{a}\right)\left|\Phi_{a}\right\rangle
\end{aligned}
$$

where $\left|\Phi_{0}\right\rangle$ is a mean-field wave function for the closedcore which we obtain by using the Dirac-Hartree-Fock (DHF) method, $T$ is the RCC operator that accounts correlation effects in terms of generating all possible CSFs from $\left|\Phi_{0}\right\rangle$ and $\left|\Phi_{a}\right\rangle=a_{a}\left|\Phi_{0}\right\rangle$ is defined as the modified reference state for the new ASF $\left|\Psi_{a}\right\rangle$.

The considered ions are highly charged systems, but the electron correlation effects in these ions are anticipated to dominate over the quantum electrodynamics (QED) interactions. However, these QED effects will be immensely large compared to the neutral atoms and the singly charged ions. For the highly charged ions, the orbitals are contracted and concentrated around the nucleus. In such case, the many-body atomic Hamiltonian can be approximated to the kinetic energies of the electrons expressed using the Dirac theory, the nuclear potential and the leading order correction terms from QED. This would be a quite reasonable choice for describing the relativistic effects in the bound electrons of the considered ions. In this work, we restrict the two-body interactions between the electrons to one-photon exchange interaction due to the longitudinal and transverse components as encapsulated in terms of the Coulomb and the approximated frequency independent Breit interactions, respectively. All together, the atomic Hamiltonian is given by

$$
\begin{aligned}
H= & \sum_{i} \Lambda_{i}^{+}\left[c \boldsymbol{\alpha}_{i} \cdot \mathbf{p}_{i}+\left(\beta_{i}-1\right) c^{2}+V_{n}\left(r_{i}\right)\right] \Lambda_{i}^{+} \\
& +\sum_{i, j>i} \Lambda_{i}^{+} \Lambda_{j}^{+} V_{e e}\left(r_{i j}\right) \Lambda_{i}^{+} \Lambda_{j}^{+}
\end{aligned}
$$

where $\boldsymbol{\alpha}_{i}$ and $\beta_{i}$ are the usual Dirac matrices and the symbol $\Lambda^{+}$ensures that when the operators act only on the positive energy states, it gives the finite values else the contributions from the negative energy states are suppressed. Subtraction of the identity operator from $\beta$ means that the energies are scaled over the rest mass energies of the electrons. We take the effective nuclear potential as $V_{n}\left(r_{i}\right)=V_{f m}\left(r_{i}\right)+V^{V P}\left(r_{i}\right)+V^{S E}\left(r_{i}\right)$ and the two-body interaction potential as $V_{e e}\left(r_{i j}\right)=$ $\frac{1}{r_{i j}}+V_{B}\left(r_{i j}\right)$. Therefore, in the Dirac-Coulomb (DC) approximation we have

$H^{D C}=\sum_{i}\left[c \boldsymbol{\alpha}_{i} \cdot \mathbf{p}_{i}+\left(\beta_{i}-1\right) c^{2}+V_{f m}\left(r_{i}\right)+\sum_{j>i} \frac{1}{r_{i j}}\right]$,

with $V_{f m}\left(r_{i}\right)$ is the nuclear Coulomb potential obtained using the Fermi charge distribution. The approximated frequency independent Breit interaction Hamiltonian is given by [41]

$$
V_{B}\left(r_{i j}\right)=-\frac{1}{2 r_{i j}}\left\{\boldsymbol{\alpha}_{i} \cdot \boldsymbol{\alpha}_{j}+\left(\boldsymbol{\alpha}_{i} \cdot \hat{\mathbf{r}}_{\mathbf{i j}}\right)\left(\boldsymbol{\alpha}_{\mathbf{j}} \cdot \hat{\mathbf{r}}_{\mathbf{i j}}\right)\right\}
$$

The leading order corrections from the vacuum polarization (VP) radiative effects is taken to be the Uehling and Wichmann-Kroll potential as $V^{V P}(r)=V_{U h l}(r)+$ $V_{W K}(r)$ [42] with

$V_{U h l}(r)=-\frac{4}{9 c \pi} V_{f m}(r) \int_{1}^{\infty} d t \sqrt{t^{2}-1}\left(\frac{1}{t^{2}}+\frac{1}{2 t^{4}}\right) e^{-2 c t r}$

and

$$
V_{W K}(r)=-\frac{2}{3} \frac{1}{c \pi} V_{f m}(r) \frac{0.092 c^{2} Z^{2}}{1+(1.62 c r)^{4}} .
$$

Similarly, the self-energy (SE) correction from the radiative effect is approximated to the contributions from 

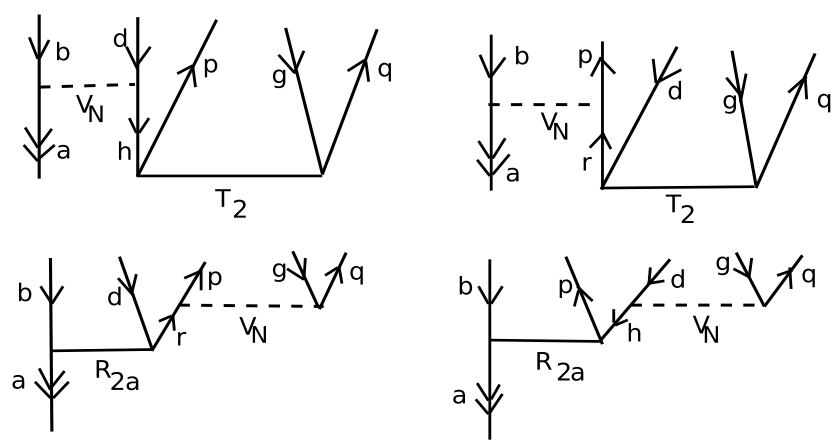

FIG. 7: Goldstone diagrams of the $R_{3 a}^{\text {pert }}$ operator that are accounted perturbatively in the $\operatorname{CCSD}(\mathrm{T})$ method.

the magnetic and electronic form factors as $V^{S E}(r)=$ $V_{m f}(r)+V_{e f}(r)[42]$ with

$$
V_{m f}(r)=-\frac{1}{4 c^{2} \pi} \gamma \cdot \nabla\left[V_{f m}(r)\left(\int_{1}^{\infty} d t \frac{e^{-2 c t r}}{\sqrt{t^{2}-1}}\right)\right]
$$

and

$$
\begin{aligned}
V_{e f}(r)= & -A(Z, r) \frac{1}{c \pi} V_{f m}(r) \int_{1}^{\infty} d t \frac{e^{-2 c t r}}{\sqrt{t^{2}-1}}\left[\left(1-\frac{1}{2 t^{2}}\right)\right. \\
& \left.\times\left\{\ln \left(t^{2}-1\right)+4 \ln (c / Z+0.5)\right\}-\frac{3}{2}+\frac{1}{t^{2}}\right] \\
& -B(Z) \frac{Z^{4}}{c^{3}} e^{-Z r},
\end{aligned}
$$

for the quantities $A(Z, r)=[1.071-1.97((Z-$ $\left.80) / c)^{2}-2.128((Z-80) / c)^{3}+0.169((Z-80) / c)^{4}\right] c r /(c r+$ $\left.0.07 Z^{2} / c^{2}\right)$ and $B(Z)=0.074+0.35 Z / c$.

In the above expressions, we have adopted au units which we shall follow-up in the rest of the paper. Also it is assumed that the mass of the nucleus is infinitely heavy. Thus, the corrections from the reduced mass of the electrons and the nuclear recoil effect, which are inversely proportional to the nuclear mass [39, 43], are neglected in the present calculations.

For the simplicity, the normal order Hamiltonian has been used in our calculations with respect to the reference state $\left|\Phi_{0}\right\rangle$ by defining

$$
H_{N}=H-\left\langle\Phi_{0}|H| \Phi_{0}\right\rangle=H-E_{S C F},
$$

with the self-consistent-field (SCF) Hartree-Fock energy $E_{S C F}$.

The amplitude solving equations for the $T$ operators for a closed-shell configuration are well known and can be referred to [44 46] for any more required explanation. We have restricted to only singly and doubly excited configurations from $\left|\Phi_{0}\right\rangle$ in our calculations (known as CCSD method) by defining $T=T_{1}+T_{2}$ which in the second quantization notation are given by

$$
T_{1}=\sum_{a, p} a_{p}^{\dagger} a_{a} t_{a}^{p}, \quad \text { and } \quad T_{2}=\frac{1}{4} \sum_{a b, p q} a_{p}^{\dagger} a_{q}^{\dagger} a_{b} a_{a} t_{a b}^{p q}
$$
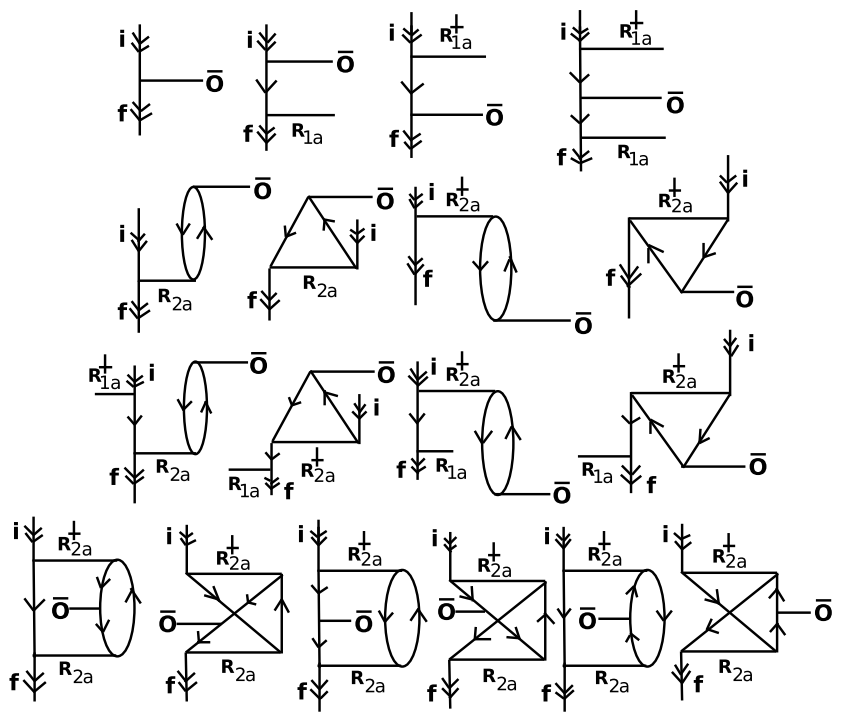

FIG. 8: Final property evaluating diagrams connecting effective one-body $\bar{O}$ operators with $R_{a}$ and its complex conjugate (cc) diagrams.

where the subscripts $a, b$ and $p, q$ represent the core and virtual orbitals, $a$ and $a^{\dagger}$ are the annihilation and creation operators, and $t_{a}^{p}$ and $t_{a b}^{p q}$ are the excitation amplitudes for the creation of the singly and doubly excited configurations.

Now the eigenvalue equations for the required states are given by

$$
\begin{aligned}
H\left|\Psi_{a}\right\rangle & =E_{a}\left|\Phi_{a}\right\rangle \\
\left(\widehat{H_{N} e^{T}}\right)_{f c}+\left(\widehat{H_{N} e^{T}}\right)_{o p} & \left.+E_{S C F}\right)\left\{1+R_{a}\right\}\left|\Phi_{a}\right\rangle \\
& =E_{a}\left\{1+R_{a}\right\}\left|\Phi_{a}\right\rangle \\
\left(\widehat{H_{N} e^{T}}\right)_{o p}\left\{1+R_{a}\right\}\left|\Phi_{a}\right\rangle & =\Delta E_{a}\left\{1+R_{a}\right\}\left|\Phi_{a}\right\rangle .
\end{aligned}
$$

The subscripts $f c$ and op represent the fully contracted and operator form of $\widehat{H_{N} e^{T}}$, with widehat symbol representing only the connecting terms, that are obtained multiplying by $e^{-T}$ from the left hand side in the above equation and $\Delta E_{a}$ refers to the electron detachment energy or ionization potential (IP) of the electron to remove it from the orbital $a$ of the $\left|\Psi_{0}\right\rangle$ state; i.e. from the ASF of the $\left[3 s^{2} 3 p^{6}\right]$ configuration. Here onwards we drop the subscript op for further discussions as the fully contracted terms will not appear any more.

We again restrict $R_{a}$ operators to account only the singly and doubly excited configurations from the corresponding $\left|\Phi_{a}\right\rangle$ reference states in order to be consistent with the CCSD method by defining $R_{a}=R_{1 a}+R_{2 a}$ which in the second quantization form are expressed as

$$
R_{1 a}=\sum_{b \neq a} a_{b}^{\dagger} a_{a} r_{a}^{b}, \text { and } R_{2 a}=\frac{1}{2} \sum_{b d, p} a_{b}^{\dagger} a_{p}^{\dagger} a_{d} a_{a} r_{a d}^{b p}
$$


TABLE I: Electron detachment energies (in $\mathrm{cm}^{-1}$ ) of few low lying states in Mn IX, Fe X, Co XI and Ni XII ions using the DHF, CCSD and CCSD(T) methods with DC, (DC+Breit), (DC+Breit+VP) and (DC+Breit+VP+SE) Hamiltonians. The results are compared with the experimental values listed in the NIST database [23]. The differences between the NIST data and our final results from the $\operatorname{CCSD}(\mathrm{T})$ method are quoted as $\delta$ in percentage (\%). We also give $y=\left(\Delta E_{a}^{D C}-\Delta E_{a}^{f i n a l}\right) / \Delta E_{a}^{\text {final }}$ values for the undertaken states in the above ions to demonstrate the trends of the relativistic effects in the heavier ions.

\begin{tabular}{|c|c|c|c|}
\hline Method & $\begin{array}{c}3 s^{2} 3 p^{5}{ }^{2} P_{3 / 2} \\
\operatorname{CCSD}(\mathrm{T})\end{array}$ & $\begin{array}{c}3 s^{2} 3 p^{5}{ }^{2} P_{1 / 2} \\
\operatorname{CCSD}(\mathrm{T})\end{array}$ & $\begin{array}{c}3 s 3 p^{62} S_{1 / 2} \\
\operatorname{CCSD}(\mathrm{T})\end{array}$ \\
\hline \multicolumn{4}{|l|}{ Mn IX } \\
\hline DHF & 1575067.58 & 1588266.78 & 1909515.85 \\
\hline $\mathrm{DC}$ & $1576861.85(-471.09)$ & $1589743.76(-490.04)$ & $1843896.78(-1167.62)$ \\
\hline+ Breit & $1576457.42(-475.16)$ & $1589006.95(-496.11)$ & $1843351.89(-1169.82)$ \\
\hline$+\mathrm{VP}$ & $1576453.12(-475.17)$ & $1589002.80(-496.11)$ & $1843377.15(-1169.76)$ \\
\hline$+\mathrm{SE}$ & $1577268.24(-472.06)$ & $1589853.50(-492.94)$ & $1844714.05(-1163.77)$ \\
\hline NIST & 1576600.01 & 1589146.02 & 1842008.01 \\
\hline$\delta(\%)$ & 0.04 & 0.04 & 0.14 \\
\hline$y$ & 0.0002 & 0.0001 & 0.0004 \\
\hline
\end{tabular}

\begin{tabular}{|c|c|c|c|}
\hline \multicolumn{4}{|l|}{$\mathrm{Fe} \mathrm{X}$} \\
\hline $\mathrm{DHF}$ & 1883771.66 & 1900218.87 & 2245974.19 \\
\hline $\mathrm{DC}$ & $1886047.50(-512.23)$ & $1902131.24(-534.99)$ & $2176829.41(-1309.54)$ \\
\hline+ Breit & $1885528.83(-518.04)$ & $1901215.38(-542.47)$ & $2176152.87(-1312.49)$ \\
\hline$+\mathrm{VP}$ & $1885523.73(-518.06)$ & $1901210.48(-542.48)$ & $2176184.13(-1312.43)$ \\
\hline$+\mathrm{SE}$ & $1886621.73(-514.53)$ & $1902361.90(-538.86)$ & $2177937.43(-1305.17)$ \\
\hline NIST & 1884000.01 & 1899683.01 & 2173249.02 \\
\hline$\delta(\%)$ & 0.14 & 0.14 & 0.21 \\
\hline$y$ & 0.0003 & 0.0001 & 0.0005 \\
\hline \multicolumn{4}{|l|}{ Co XI } \\
\hline DHF & 2217435.11 & 2237682.21 & 2608011.58 \\
\hline DC & $2219848.89(-509.21)$ & $2239675.97(-535.29)$ & $2534877.86(-1457.54)$ \\
\hline +Breit & $2219196.01(-515.14)$ & $2238552.11(-542.92)$ & $2534047.65(-1460.75)$ \\
\hline$+\mathrm{VP}$ & $2219190.05(-515.15)$ & $2238546.42(-542.94)$ & $2534085.85(-1460.71)$ \\
\hline$+\mathrm{SE}$ & $2220636.28(-511.12)$ & $2240070.27(-538.77)$ & $2536349.04(-1451.66)$ \\
\hline NIST & 2221000.01 & 2240345.02 & 2534630.02 \\
\hline$\delta(\%)$ & 0.02 & 0.01 & 0.06 \\
\hline$y$ & 0.0003 & 0.0002 & 0.0006 \\
\hline \multicolumn{4}{|l|}{ Ni XII } \\
\hline DHF & 2575996.48 & 2600654.35 & 2995633.72 \\
\hline $\mathrm{DC}$ & $2579954.22(-467.87)$ & $2604148.24(-496.73)$ & $2919968.40(-1496.84)$ \\
\hline+ Breit & $2578738.04(-605.35)$ & $2602370.96(-637.96)$ & $2918845.93(-1485.21)$ \\
\hline$+\mathrm{VP}$ & $2578731.71(-604.76)$ & $2602365.04(-637.34)$ & $2918893.03(-1484.39)$ \\
\hline$+\mathrm{SE}$ & $2580601.25(-600.73)$ & $2604345.11(-632.42)$ & $2921767.82(-1474.02)$ \\
\hline NIST & 2577200.01 & 2600829.02 & 2915815.02 \\
\hline$\delta(\%)$ & 0.13 & 0.13 & 0.20 \\
\hline$y$ & 0.0002 & 0.0001 & 0.0006 \\
\hline
\end{tabular}

where the sum for $R_{2 a}$ includes $b=a$ without any loss of generality to facilitate the simple angular momentum algebra at the cost of violating the Pauli's exclusion principle. The extra contributions anticipated from these unphysical contributions are, however, cancel out from the direct and exchange parts of the two-body diagrams by allowing contributions only from the linked diagrams in the calculations [47]. Goldstone diagrammatical interpre- tation of the $T_{1}, T_{2}, R_{1 a}$ and $R_{2 a}$ operators are shown in Fig. 11

The energy and amplitude solving equations for the $R_{a}$ wave operators are given by

$$
\begin{aligned}
\left\langle\Phi_{a}\left|\widehat{H_{N} e^{T}}\left\{1+R_{a}\right\}\right| \Phi_{a}\right\rangle & =\Delta E_{a} \\
\left.\left\langle\Phi_{a}^{b}\right| \widehat{\left(H_{N} e^{T}\right.}-\Delta E_{a}\right) R_{a}\left|\Phi_{a}\right\rangle & =-\left\langle\Phi_{a}^{b}\left|\widehat{H_{N} e^{T}}\right| \Phi_{a}\right\rangle
\end{aligned}
$$




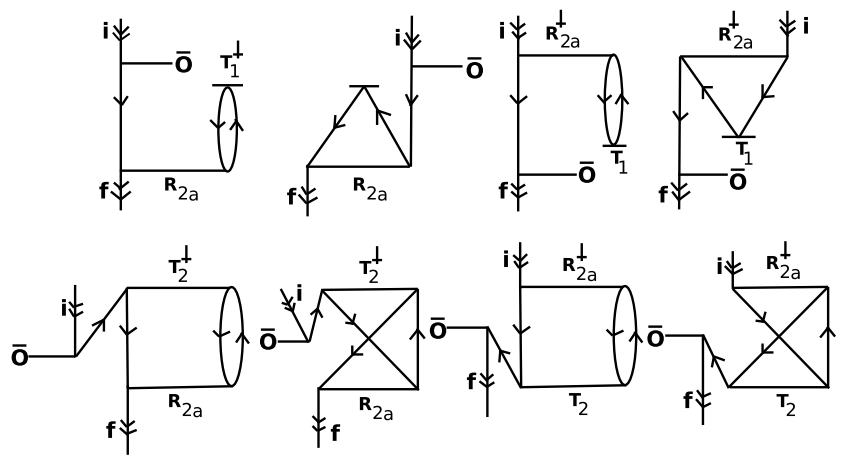

FIG. 9: Few important diagrams connecting effective twobody $\bar{O}$ operators with $R_{a}$ and its $c c$ diagrams that are contributing significantly in the transition amplitude calculations.

and

$$
\left\langle\Phi_{d a}^{p b}\left|\left(\widehat{H_{N} e^{T}}-\Delta E_{a}\right) R_{a}\right| \Phi_{a}\right\rangle=-\left\langle\Phi_{d a}^{p b}\left|\widehat{H_{N} e^{T}}\right| \Phi_{a}\right\rangle,
$$

where $\left|\Phi_{a}^{b}\right\rangle$ are the singly excited configurations from $\left|\Phi_{a}\right\rangle$ constructed as replacing orbital $a$ by orbital $b$ and $\left|\Phi_{d a}^{p b}\right\rangle$ denotes doubly excited configurations from $\left|\Phi_{a}\right\rangle$ constructed as replacing orbital $a$ by orbital $b$ along with exciting an electron from the occupied orbital $d$ to virtual orbital $p$ as per the definitions given in Eq. (27). The above non-linear equations are solved self-consistently along with its energy evaluating equation.

We take the help of diagrammatic representation to get solutions in an easier way for the above equations. In this process, we divide first as $H_{N}=F_{N}+V_{N}$ with $F_{N}$ representing the DHF Hamiltonian which is an effective one-body operator and $V_{N}$ is the normal ordering form of the residual Coulomb-Breit interaction. By construction, $F_{N}$ is diagonal in nature and the one-body contributions from $V_{N}$ cancels out in our calculations. Following the Koopman's theorem [47], the detachment energy of an electron from orbital $a$ at the DHF level is just the diagonal value of the operator $F_{N}$ (the single particle orbital energy of $a$ ). To minimize the computational time, we construct effective one-body and two-body intermediate terms from $\widehat{F_{N} e^{T}}$ and $\widehat{V_{N} e^{T}}$ as shown in Figs. 2 and 3 , respectively, and connect them finally with the $R_{a}$ operators to solve the above energy and amplitude equations. The energy evaluating diagrams are shown in Fig. 4. Similarly, the diagrams contributing to the $R_{1 a}$ and $R_{2 a}$ amplitude calculations are shown in Figs. 5 and 6 , respectively.

The quality of the results are further elevated with the consideration of the most important triple excitation configurations from $\left|\Phi_{a}\right\rangle$ by constructing a perturbative RCC operator $R_{3 a}^{\text {pert }}$ as

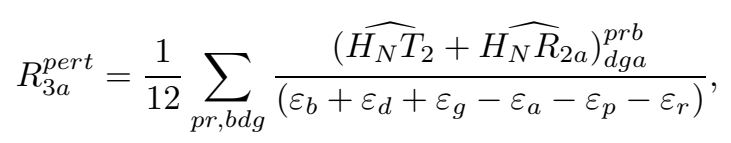

TABLE II: Sensitivity coefficients $q$ (in $\mathrm{cm}^{-1}$ ) for the first three low-lying transitions in Mn IX, Fe X, Co XI and Ni XII ions from the $\operatorname{CCSD}(\mathrm{T})$ method using the $\mathrm{DC}$, (DC+Breit),

\begin{tabular}{|c|c|c|c|}
\hline Method & $\begin{array}{c}3 s^{2} 3 p^{5}{ }^{2} P_{3 / 2} \rightarrow \\
3 s 3 p^{6}{ }^{2} S_{1 / 2} \\
\end{array}$ & $\begin{array}{c}3 s^{2} 3 p^{5}{ }^{2} P_{1 / 2} \rightarrow \\
3 s 3 p^{6}{ }^{2} S_{1 / 2} \\
\end{array}$ & $\begin{array}{c}3 s^{2} 3 p^{5}{ }^{2} P_{3 / 2} \rightarrow \\
3 s^{2} 3 p^{5}{ }^{2} P_{1 / 2} \\
\end{array}$ \\
\hline \multicolumn{4}{|l|}{ Mn IX } \\
\hline DHF & 18387.57 & 4934.39 & 13444.18 \\
\hline $\mathrm{DC}$ & 16120.40 & 3014.10 & 13105.50 \\
\hline+ Breit & 15933.81 & 3160.00 & 12772.97 \\
\hline$+\mathrm{VP}$ & 15977.78 & 3204.45 & 12773.33 \\
\hline$+\mathrm{SE}$ & 16849.90 & 3984.50 & 12865.40 \\
\hline \multicolumn{4}{|l|}{ Fe X } \\
\hline DHF & 22613.10 & 5832.88 & 16780.22 \\
\hline DC & 19898.70 & 3505.90 & 16392.70 \\
\hline+ Breit & 19694.16 & 3698.94 & 15995.22 \\
\hline$+\mathrm{VP}$ & 19748.35 & 3752.61 & 15995.74 \\
\hline$+\mathrm{SE}$ & 20834.87 & 4703.69 & 16131.18 \\
\hline \multicolumn{4}{|l|}{ Co XI } \\
\hline DHF & 27516.40 & 6823.61 & 20692.78 \\
\hline $\mathrm{DC}$ & 24273.10 & 4026.20 & 20246.90 \\
\hline+ Breit & 24050.83 & 4276.94 & 19773.89 \\
\hline$+\mathrm{VP}$ & 24116.81 & 4342.19 & 19774.62 \\
\hline$+\mathrm{SE}$ & 25458.00 & 5488.80 & 19969.20 \\
\hline DHF & 33167.97 & 7921.84 & 25246.13 \\
\hline \multicolumn{4}{|l|}{ Ni XII } \\
\hline DC & 29338.30 & 4594.00 & 24746.20 \\
\hline+ Breit & 29101.08 & 4909.73 & 24191.34 \\
\hline$+\mathrm{VP}$ & 29180.79 & 4988.45 & 24192.34 \\
\hline$+\mathrm{SE}$ & 30828.80 & 6363.90 & 24464.90 \\
\hline
\end{tabular}
$(\mathrm{DC}+$ Breit $+\mathrm{VP})$ and $(\mathrm{DC}+$ Breit $+\mathrm{VP}+\mathrm{SE})$ Hamiltonians.

with $\varepsilon$ s representing the single particle orbital energies. Instead of considering this operator explicitly, we account its contributions implicitly in the self-consistent evaluation of $\Delta E_{a}$. This approach is usually referred to as $\operatorname{CCSD}(\mathrm{T})$ method in the literature. Diagrammatic representation of the $R_{3 a}^{\text {pert }}$ operator are given in Fig. 7

We evaluate $\Delta E_{a} \mathrm{~s}$ using the laboratory value as $c=$ 137.03599972 and modify the $c$ value suitably for the corresponding $x$ values to obtain the transition frequencies, $\omega(x)$, between all possible states that are of our interest.

Once any two given $\left|\Psi_{f}\right\rangle$ and $\left|\Psi_{i}\right\rangle$ states are obtained in the above procedure, the matrix element of an operator $O$ between these two states are evaluated using the expression

$$
\frac{\left\langle\Psi_{f}|O| \Psi_{i}\right\rangle}{\sqrt{\left\langle\Psi_{f} \mid \Psi_{f}\right\rangle\left\langle\Psi_{i} \mid \Psi_{i}\right\rangle}}=\frac{\left\langle\Phi_{f}\left|\left\{1+R_{f}^{\dagger}\right\} \bar{O}\left\{1+R_{i}\right\}\right| \Phi_{i}\right\rangle}{\sqrt{\mathcal{N}_{f} \mathcal{N}_{i}}}
$$

where $\bar{O}=\left(e^{T^{\dagger}} O e^{T}\right)_{l}$ and $\mathcal{N}_{i}=\left\{\left(1+R_{i}^{\dagger}\right) \overline{\mathcal{N}}\left(1+R_{i}\right)\right\}$ with $\overline{\mathcal{N}}=\left(e^{T^{\dagger}} e^{T}\right)_{l}$, for the subscript $l$ means only the linked terms are the contributing terms, involves two non-truncative series in the above expression whose con- 
TABLE III: E1, M1 and E2 matrix elements (in au) for the considered transitions in the Mn IX, Fe X, Co XI and Ni XII ions coming from the DHF, DC, (DC+Breit), $(\mathrm{DC}+$ Breit $+\mathrm{VP})$ and $(\mathrm{DC}+$ Breit $+\mathrm{VP}+\mathrm{SE})$ approximations. We have given these results from the CCSD method and contributions from the partial triple excitations are added at the end. The recommended values from our calculations with uncertainties are quoted as "Reco".

\begin{tabular}{|c|c|c|c|c|}
\hline \multirow[t]{2}{*}{ Method } & \multicolumn{2}{|c|}{$\begin{array}{c}3 s^{2} 3 p^{5}{ }^{2} P_{1 / 2} \rightarrow \\
3 s^{2} 3 p^{5}{ }^{2} P_{3 / 2} \\
\end{array}$} & \multirow{2}{*}{$\begin{array}{c}3 s 3 p^{6}{ }^{2} S_{1 / 2} \rightarrow \\
3 s^{2} 3 p^{5}{ }^{2} P_{1 / 2} \\
\text { E1 }\end{array}$} & \multirow{2}{*}{$\begin{array}{c}3 s 3 p^{6}{ }^{2} S_{1 / 2} \rightarrow \\
3 s^{2} 3 p^{5}{ }^{2} P_{3 / 2} \\
\text { E1 }\end{array}$} \\
\hline & M1 & E2 & & \\
\hline \multicolumn{5}{|l|}{ Mn IX } \\
\hline DHF & 1.15421 & 0.68517 & 0.65197 & 0.92508 \\
\hline DC & 1.15082 & 0.59500 & 0.35195 & 0.40368 \\
\hline+ Breit & 1.15079 & 0.59540 & 0.35163 & 0.40330 \\
\hline$+\mathrm{VP}$ & 1.15080 & 0.59540 & 0.35160 & 0.40324 \\
\hline$+\mathrm{SE}$ & 1.15080 & 0.59483 & 0.35211 & 0.40455 \\
\hline +Triples & 1.15078 & 0.59486 & 0.35316 & 0.40660 \\
\hline Reco & $1.1508(1)$ & $0.5948(4)$ & $0.353(1)$ & $0.406(1)$ \\
\hline \multicolumn{5}{|l|}{$\mathrm{Fe} X$} \\
\hline DHF & 1.15414 & 0.59914 & 0.61202 & 0.86869 \\
\hline $\mathrm{DC}$ & 1.15187 & 0.52127 & 0.33404 & 0.38622 \\
\hline+ Breit & 1.15185 & 0.52177 & 0.33475 & 0.38783 \\
\hline$+\mathrm{VP}$ & 1.15184 & 0.52172 & 0.33369 & 0.38576 \\
\hline$+\mathrm{SE}$ & 1.15185 & 0.52133 & 0.33438 & 0.38725 \\
\hline +Triples & 1.15183 & 0.52135 & 0.33529 & 0.38923 \\
\hline Reco & $1.1518(1)$ & $0.5213(2)$ & $0.335(1)$ & $0.389(1)$ \\
\hline \multicolumn{5}{|l|}{ Co XI } \\
\hline DHF & 1.15406 & 0.52867 & 0.57670 & 0.81887 \\
\hline $\mathrm{DC}$ & 1.15257 & 0.46292 & 0.31750 & 0.36755 \\
\hline+ Breit & 1.15254 & 0.46335 & 0.31714 & 0.36717 \\
\hline$+\mathrm{VP}$ & 1.15253 & 0.46336 & 0.31710 & 0.36711 \\
\hline$+\mathrm{SE}$ & 1.15255 & 0.46386 & 0.31768 & 0.36877 \\
\hline +Triples & 1.15253 & 0.46398 & 0.31866 & 0.37071 \\
\hline Reco & $1.1525(1)$ & $0.464(1)$ & $0.319(1)$ & $0.370(1)$ \\
\hline \multicolumn{5}{|l|}{ Ni XII } \\
\hline DHF & 1.15397 & 0.47016 & 0.54525 & 0.77451 \\
\hline $\mathrm{DC}$ & 1.15214 & 0.43969 & 0.28383 & 0.34649 \\
\hline+ Breit & 1.15243 & 0.41536 & 0.30295 & 0.35314 \\
\hline$+\mathrm{VP}$ & 1.15243 & 0.41538 & 0.30292 & 0.35307 \\
\hline$+\mathrm{SE}$ & 1.15250 & 0.41472 & 0.30392 & 0.35480 \\
\hline +Triples & 1.15250 & 0.41473 & 0.30479 & 0.35653 \\
\hline Reco & $1.1525(2)$ & $0.4147(1)$ & $0.3048(3)$ & $0.3565(1)$ \\
\hline
\end{tabular}

tributions are accounted as much as possible in stepwise. To do so, we divide $\bar{O}$ and $\overline{\mathcal{N}}$ into the effective fully contracted, one-body, two-body etc. terms in the diagrammatic form by employing the generalized Wick's theorem [47]. Since these terms are either connected with the $R_{a}$ operators or has to be the effective one-body term for the consideration at the final stage property calculation,
TABLE IV: Contributions to the E1, M1 and E2 amplitudes from various $\mathrm{RCC}$ terms of the $\mathrm{CCSD}(\mathrm{T})$ method using the $(\mathrm{DC}+$ Breit $+\mathrm{VP}+\mathrm{SE})$ Hamiltonian in the Mn IX and Fe X ions.

\begin{tabular}{|c|c|c|c|c|}
\hline \multirow[t]{2}{*}{$\begin{array}{l}\mathrm{RCC} \\
\text { term }\end{array}$} & \multicolumn{2}{|c|}{$\begin{array}{c}3 s^{2} 3 p^{5}{ }^{2} P_{1 / 2} \rightarrow \\
3 s^{2} 3 p^{5}{ }^{2} P_{3 / 2} \\
\end{array}$} & \multirow{2}{*}{$\begin{array}{c}3 s 3 p^{6}{ }^{2} S_{1 / 2} \rightarrow \\
3 s^{2} 3 p^{5}{ }^{2} P_{1 / 2} \\
\text { E1 }\end{array}$} & \multirow{2}{*}{$\begin{array}{c}3 s 3 p^{6}{ }^{2} S_{1 / 2} \rightarrow \\
3 s^{2} 3 p^{5}{ }^{2} P_{3 / 2} \\
\text { E1 } \\
\end{array}$} \\
\hline & M1 & $\mathrm{E} 2$ & & \\
\hline \multicolumn{5}{|l|}{ Mn IX } \\
\hline $\bar{O}_{o b}$ & -1.14501 & -0.66667 & 0.64321 & -0.91119 \\
\hline $\bar{O}_{o b} R_{1 a}$ & 0.00001 & 0.00006 & -0.00005 & 0.00008 \\
\hline$R_{1 a}^{\dagger} \bar{O}$ & 0.00001 & 0.00006 & 0.00006 & -0.00009 \\
\hline $\bar{O}_{o b} R_{2 a}$ & 0.00039 & 0.03221 & -0.09488 & 0.13285 \\
\hline$R_{2 a}^{\dagger} \bar{O}$ & -0.00030 & 0.03211 & -0.22270 & 0.32237 \\
\hline$R_{1 a}^{\dagger} \bar{O}_{o b} R_{1 a}$ & $\sim 0$ & $\sim 0$ & $\sim 0$ & $\sim 0$ \\
\hline$R_{1 a}^{\dagger} \bar{O}_{o b} R_{2 a}$ & $\sim 0$ & $\sim 0$ & $\sim 0$ & $\sim 0$ \\
\hline$R_{2 a}^{\dagger} \bar{O}_{o b} R_{1 a}$ & $\sim 0$ & $\sim 0$ & $\sim 0$ & $\sim 0$ \\
\hline$R_{2 a}^{\dagger} \bar{O}_{o b} R_{2 a}$ & -0.01899 & 0.00323 & 0.02624 & 0.05171 \\
\hline Others & -0.00094 & -0.00313 & 0.00278 & -0.00400 \\
\hline norm & 0.01408 & 0.00727 & -0.00150 & 0.00167 \\
\hline \multicolumn{5}{|l|}{$\mathrm{Fe} X$} \\
\hline $\bar{O}_{o b}$ & -1.14547 & -0.58110 & 0.60414 & -0.85686 \\
\hline $\bar{O}_{o b} R_{1 a}$ & 0.00001 & 0.00005 & -0.00004 & 0.00007 \\
\hline$R_{1 a}^{\dagger} \bar{O}$ & -0.00001 & 0.00004 & 0.00007 & -0.00010 \\
\hline $\bar{O}_{o b} R_{2 a}$ & 0.00039 & 0.02664 & -0.08733 & 0.12207 \\
\hline$R_{2 a}^{\dagger} \bar{O}$ & -0.00030 & 0.02658 & -0.20625 & 0.30004 \\
\hline$R_{1 a}^{\dagger} \bar{O}_{o b} R_{1 a}$ & $\sim 0$ & $\sim 0$ & $\sim 0$ & $\sim 0$ \\
\hline$R_{1 a}^{\dagger} \bar{O}_{o b} R_{2 a}$ & $\sim 0$ & $\sim 0$ & $\sim 0$ & $\sim 0$ \\
\hline$R_{2 a}^{\dagger} \bar{O}_{o b} R_{1 a}$ & $\sim 0$ & $\sim 0$ & $\sim 0$ & $\sim 0$ \\
\hline$R_{2 a}^{\dagger} \bar{O}_{o b} R_{2 a}$ & -0.01925 & 0.00268 & 0.02369 & 0.04742 \\
\hline Others & -0.00080 & -0.00237 & 0.00237 & -0.00340 \\
\hline norm & 0.01362 & 0.00613 & -0.00136 & 0.00153 \\
\hline
\end{tabular}

as a result they get truncated factitiously at the effective five-body terms in the CCSD method. The intermediate storage of the effective three-body terms onwards is an affair of huge computational cost and direct calculation of these diagrams will be enormously time consuming against a very little contribution to the final result. Therefore, we have neglected these contributions on the basis that they belong to the class of diagrams with fifth or higher orders in the residual Coulomb-Breit interaction. We first calculate the intermediate effective one-body diagrams of hole-hole $(\mathrm{H}-\mathrm{H})$, particle-particle $(\mathrm{P}-\mathrm{P})$, hole-particle (H-P) and particle-hole (P-H) types from $\bar{O}$ and $\overline{\mathcal{N}}$ considering terms up to minimum fifth order in the residual Coulomb-Breit interaction and store these intermediate parts for their further use. It has been found in our study, as will be demonstrated in the next Results and Discussion section, that the H-P and $\mathrm{P}-\mathrm{H}$ diagrams carrying out the core-polarization effects to all orders are contributing predominantly in the considered ions. Therefore, we have replaced the corresponding $O$ operator from the $\mathrm{P}-\mathrm{H}$ and $\mathrm{H}-\mathrm{P}$ effective diagrams by the $\mathrm{P}-\mathrm{P}$ and $\mathrm{H}-\mathrm{H}$ diagrams to dress-up further the effective 
TABLE V: Contributions to the E1, M1 and E2 amplitudes from various RCC terms of the $\operatorname{CCSD}(\mathrm{T})$ method using the (DC+Breit+VP+SE) Hamiltonian in the Co XI and Ni XII ions.

\begin{tabular}{lcccc}
\hline \hline RCC & $3 s^{2} 3 p^{5}{ }^{2} P_{1 / 2} \rightarrow$ & $3 s 3 p^{6}{ }^{2} S_{1 / 2} \rightarrow$ & $3 s 3 p^{6}{ }^{2} S_{1 / 2} \rightarrow$ \\
term & \begin{tabular}{c}
$3 s^{2} 3 p^{5}{ }^{2} P_{3 / 2}$ \\
\cline { 2 - 2 }
\end{tabular} & $3 s^{2} 3 p^{5}{ }^{2} P_{1 / 2}$ & $3 s^{2} 3 p^{5}{ }^{2} P_{3 / 2}$ \\
& M1 & E2 & E1 & E1 \\
\hline
\end{tabular}

\begin{tabular}{|c|c|c|c|c|}
\hline \multicolumn{5}{|l|}{ Co XI } \\
\hline $\bar{O}_{o b}$ & -1.14578 & -0.51431 & 0.56952 & -0.80751 \\
\hline $\bar{O}_{o b} R_{1 a}$ & 0.00001 & 0.00004 & -0.00004 & 0.00006 \\
\hline$R_{1 a}^{\dagger} \bar{O}$ & -0.00001 & 0.00004 & 0.00007 & -0.00010 \\
\hline $\bar{O}_{o b} R_{2 a}$ & 0.00040 & 0.02233 & -0.08086 & 0.11282 \\
\hline$R_{2 a}^{\dagger} \bar{O}$ & -0.00030 & 0.02232 & -0.19250 & 0.28160 \\
\hline$R_{1 a}^{\dagger} \bar{O}_{o b} R_{1 a}$ & $\sim 0$ & $\sim 0$ & $\sim 0$ & $\sim 0$ \\
\hline$R_{1 a}^{\dagger} \bar{O}_{o b} R_{2 a}$ & $\sim 0$ & $\sim 0$ & $\sim 0$ & $\sim 0$ \\
\hline$R_{2 a}^{\dagger} \bar{O}_{o b} R_{1 a}$ & $\sim 0$ & $\sim 0$ & $\sim 0$ & $\sim 0$ \\
\hline$R_{2 a}^{\dagger} \bar{O}_{o b} R_{2 a}$ & -0.01930 & 0.00226 & 0.02166 & 0.04392 \\
\hline Others & -0.00069 & -0.00193 & 0.00204 & -0.00290 \\
\hline norm & 0.01316 & 0.00527 & -0.00123 & 0.00140 \\
\hline \multicolumn{5}{|l|}{ Ni XII } \\
\hline $\bar{O}_{o b}$ & -1.14539 & -0.45742 & 0.53861 & -0.76408 \\
\hline $\bar{O}_{o b} R_{1 a}$ & 0.00001 & 0.00003 & -0.00003 & 0.00005 \\
\hline$R_{1 a}^{\dagger} \bar{O}$ & -0.00001 & 0.00003 & 0.00008 & -0.00011 \\
\hline $\bar{O}_{o b} R_{2 a}$ & 0.00041 & 0.01880 & -0.07501 & 0.10445 \\
\hline$R_{2 a}^{\dagger} \bar{O}$ & -0.00030 & 0.01882 & -0.17924 & 0.26391 \\
\hline$R_{1 a}^{\dagger} \bar{O}_{o b} R_{1 a}$ & $\sim 0$ & $\sim 0$ & $\sim 0$ & $\sim 0$ \\
\hline$R_{1 a}^{\dagger} \bar{O}_{o b} R_{2 a}$ & $\sim 0$ & $\sim 0$ & $\sim 0$ & $\sim 0$ \\
\hline$R_{2 a}^{\dagger} \bar{O}_{o b} R_{1 a}$ & $\sim 0$ & $\sim 0$ & $\sim 0$ & $\sim 0$ \\
\hline$R_{2 a}^{\dagger} \bar{O}_{o b} R_{2 a}$ & -0.01917 & 0.00191 & 0.01975 & 0.04050 \\
\hline Others & -0.00069 & -0.00146 & 0.00176 & -0.00255 \\
\hline norm & 0.01274 & 0.00456 & -0.00113 & 0.00130 \\
\hline
\end{tabular}

$\mathrm{H}-\mathrm{P} / \mathrm{P}-\mathrm{H}$ operators for accounting these contributions as rigorously as possible. All these four types of effective one-body terms are then connected with the $R_{a}$ and its complex-conjugate $(c c)$ diagrams to obtain results for the final calculation. These final contributing diagrams are shown in Fig. 8.

We then formulate the effective two-body terms from $\bar{O}$ and $\overline{\mathcal{N}}$ in the following way to account their contributions at the minimum computational requirements. We connect the effective one-body terms of $\bar{O}$ with another $T$ and with its $c c$ operators to form the effective twobody diagrams. This procedure obviously takes into account more higher order terms than the two-body terms that could have been generated by connecting only the operator $O$ with the $T$ operators. Unlike the effective one-body terms, effective two-body terms are computed directly after contracting with the $R_{a}$ operators. Some of the important effective two-body diagrams contributing substantially in the present calculations are shown in Fig. 9.

Contribution to the matrix element after the normalizations of the wave functions (norm) is estimated ex-
TABLE VI: Transition rates $\left(A\right.$ in $\left.s^{-1}\right)$ and oscillator strengths $(f)$ in the considered ions.

\begin{tabular}{lcc}
\hline \hline Transition & $f_{k i}$ \\
\cline { 2 - 3 } & $\begin{array}{c}\text { Others } \\
\text { Othesent }\end{array}$ & \\
\hline
\end{tabular}

\begin{tabular}{|c|c|c|c|}
\hline & & & \\
\hline $3 s^{2} 3 p^{5}{ }^{2} P_{1 / 2} \stackrel{M 1}{\longrightarrow} 3 s^{2} 3 p^{5}{ }^{2} P_{3 / 2}$ & ${ }^{a} 35.5$ & $35.27(1)$ & $1.67(1)[-7]$ \\
\hline $3 s^{2} 3 p^{5}{ }^{2} P_{1 / 2} \stackrel{E 2}{\longrightarrow} 3 s^{2} 3 p^{5}{ }^{2} P_{3 / 2}$ & & $6.16(1)[-3]$ & $2.93(1)[-11]$ \\
\hline $3 s 3 p^{6}{ }^{2} S_{1 / 2} \stackrel{E 1}{\longrightarrow} 3 s^{2} 3 p^{5}{ }^{2} P_{1 / 2}$ & & $2.04(1)[9]$ & $4.76(3)[-2]$ \\
\hline $3 s 3 p^{6}{ }^{2} S_{1 / 2} \stackrel{E 1}{\longrightarrow} 3 s^{2} 3 p^{5}{ }^{2} P_{3 / 2}$ & & $3.13(1)[9]$ & $3.31(2)[-2]$ \\
\hline
\end{tabular}

Fe X

\begin{tabular}{lllll}
$3 s^{2} 3 p^{5}{ }^{2} P_{1 / 2} \stackrel{M 1}{\longrightarrow} 3 s^{2} 3 p^{5}{ }^{2} P_{3 / 2}$ & ${ }^{a} 69.4$ & $69.01(1)$ & $2.10(2)[-7]$ \\
& ${ }^{b} 70.4$ & & \\
$3 s^{2} 3 p^{5}{ }^{2} P_{1 / 2} \stackrel{E 2}{\longrightarrow} 3 s^{2} 3 p^{5}{ }^{2} P_{3 / 2}$ & & $1.44(1)[-2]$ & $4.41(1)[-11]$ \\
$3 s 3 p^{6}{ }^{2} S_{1 / 2} \stackrel{E 1}{\longrightarrow} 3 s^{2} 3 p^{5}{ }^{2} P_{1 / 2}$ & & $2.33(1)[9]$ & $4.64(3)[-2]$ \\
$3 s 3 p^{6}{ }^{2} S_{1 / 2} \stackrel{E 1}{\longrightarrow} 3 s^{2} 3 p^{5}{ }^{2} P_{3 / 2}$ & & $3.71(2)[9]$ & $3.31(2)[-2]$ \\
\hline
\end{tabular}

Co XI

$\begin{array}{lllll}3 s^{2} 3 p^{5}{ }^{2} P_{1 / 2} \stackrel{M 1}{\longrightarrow} 3 s^{2} 3 p^{5}{ }^{2} P_{3 / 2} & { }^{a} 130.0 & 129.68(2) & 2.60(1)[-7] \\ 3 s^{2} 3 p^{5}{ }^{2} P_{1 / 2} \stackrel{E 2}{\longrightarrow} 3 s^{2} 3 p^{5}{ }^{2} P_{3 / 2} & & 3.26(1)[-2] & 6.54(1)[-11] \\ 3 s 3 p^{6}{ }^{2} S_{1 / 2} \stackrel{E 1}{\longrightarrow} 3 s^{2} 3 p^{5}{ }^{2} P_{1 / 2} & & 2.62(2)[9] & 4.51(3)[-2] \\ 3 s 3 p^{6}{ }^{2} S_{1 / 2} \stackrel{E 1}{\longrightarrow} 3 s^{2} 3 p^{5}{ }^{2} P_{3 / 2} & 4.29(2)[9] & 3.25(2)[-2]\end{array}$

$\mathrm{Ni}$ XII

\begin{tabular}{llll}
$3 s^{2} 3 p^{5}{ }^{2} P_{1 / 2} \stackrel{M 1}{\longrightarrow} 3 s^{2} 3 p^{5}{ }^{2} P_{3 / 2}$ & ${ }^{a} 237.0$ & $236.31(3)$ & $3.17(1)[-7]$ \\
$3 s^{2} 3 p^{5}{ }^{2} P_{1 / 2} \stackrel{E 2}{\longrightarrow} 3 s^{2} 3 p^{5}{ }^{2} P_{3 / 2}$ & & $7.10(1)[-2]$ & $9.53(1)[-11]$ \\
$3 s 3 p^{6}{ }^{2} S_{1 / 2} \stackrel{E 1}{\longrightarrow} 3 s^{2} 3 p^{5}{ }^{2} P_{1 / 2}$ & $2.94(1)[9]$ & $4.42(1)[-2]$ \\
$3 s 3 p^{6}{ }^{2} S_{1 / 2} \stackrel{E 1}{\longrightarrow} 3 s^{2} 3 p^{5}{ }^{2} P_{3 / 2}$ & $4.99(2)[9]$ & $3.24(1)[-2]$ \\
\hline \hline
\end{tabular}

plicitly using the expression

$$
\begin{aligned}
\text { norm } & =\left[\frac{\left\langle\Psi_{f}|O| \Psi_{i}\right\rangle}{\sqrt{\left\langle\Psi_{f} \mid \Psi_{f}\right\rangle\left\langle\Psi_{i} \mid \Psi_{i}\right\rangle}}-\left\langle\Psi_{f}|O| \Psi_{i}\right\rangle\right] \\
& =\left[\frac{1}{\sqrt{\mathcal{N}_{f} \mathcal{N}_{i}}}-1\right]\left\langle\Psi_{f}|O| \Psi_{i}\right\rangle
\end{aligned}
$$

\section{RESULTS AND DISCUSSION}

We present the detachment energies obtained using our above described methods in Table I at the various levels of approximations in the Hamiltonian for all the considered ions. We give contributions from DHF and $\operatorname{CCSD}(\mathrm{T})$ results with gradual changes in the calculated values after the inclusion of Breit, VP and SE interactions. We also give the differences between the $\operatorname{CCSD}(\mathrm{T})$ and CCSD results within the parentheses of the given $\operatorname{CCSD}(\mathrm{T})$ results to demonstrate the importance of including the triple excitation configurations. It 
TABLE VII: Lifetimes $(\tau)$ of the first two excited states (in $m s)$ of the considered ions.

\begin{tabular}{lccc}
\hline \hline State & This work & $\begin{array}{c}\text { Other } \\
\text { prediction }\end{array}$ & Experiment \\
\hline
\end{tabular}

$\begin{array}{lc}\text { Mn IX } & \\ 3 s^{2} 3 p^{5}{ }^{3} P_{1 / 2} & 28.34(2) \\ 3 s 3 p^{6}{ }^{2} S_{1 / 2} & 1.93(3)[-7]\end{array}$

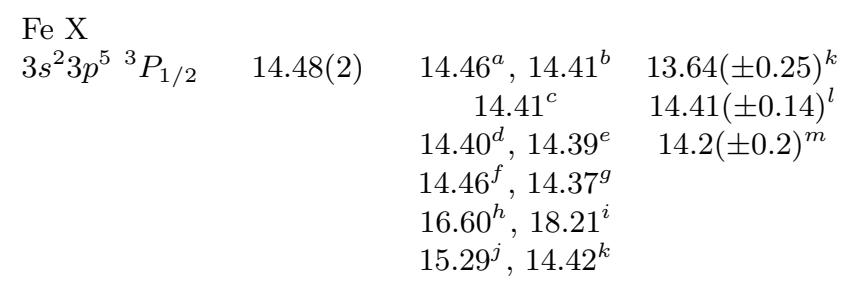

$\begin{array}{lll}3 s 3 p^{6}{ }^{2} S_{1 / 2} & 1.65(3)[-7]\end{array}$

\begin{tabular}{lccc} 
Co XI & & & \\
$3 s^{2} 3 p^{5}{ }^{3} P_{1 / 2}$ & $7.71(2)$ & $7.69^{b}, 7.69^{c}$ & $7.62( \pm 0.46)^{l}$ \\
& & $8.67^{h}$ & \\
$3 s 3 p^{6}{ }^{2} S_{1 / 2}$ & $1.44(3)[-7]$ & & \\
\hline
\end{tabular}

\begin{tabular}{|c|c|c|c|}
\hline $\begin{array}{l}\text { Ni XII } \\
3 s^{2} 3 p^{5}{ }^{3} P_{1 / 2} \\
3 s 3 p^{6}{ }^{2} S_{1 / 2}\end{array}$ & $\begin{array}{c}4.23(2) \\
1.26(3)[-7]\end{array}$ & $\begin{array}{c}4.22^{b, c, d, g} \\
4.69^{h}\end{array}$ & $4.166( \pm 0.06)^{l}$ \\
\hline & References & 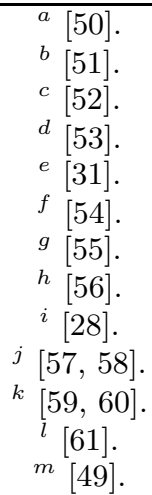 & \\
\hline
\end{tabular}

can also be noticed here that the $\operatorname{CCSD}(\mathrm{T})$ method improves the results over the CCSD approach in all the states. Our results are also compared with experimental values listed in the national institute of science and technology (NIST) database [23]. As can be seen, contributions from the higher order relativistic corrections are not small in the evaluation of these quantities. Among them the SE interaction are the largest contributing relativistic corrections. The differences between our final results with the full Hamiltonian and NIST results are given as $\delta$ in the same table which shows that the $\operatorname{CCSD}(\mathrm{T})$ results are sub-one percent accurate for each state in all the four ions. We also observe that the ratios $y=\left(\Delta E_{a}^{D C}-\Delta E_{a}^{f i n a l}\right) / \Delta E_{a}^{f i n a l}$, with $\Delta E_{a}^{D C}$ is the contribution from the DC Hamiltonian and $\Delta E_{a}^{f i n a l}$ is the final result, are almost same in all the states except in the excited $3 s^{2} 3 p^{5}{ }^{2} P_{1 / 2}$ state where it is slightly large. These values are comparatively larger in the Ni XII ion implying that the relativistic effects are increasing with the size of the ion.

The accuracies attained in the energy calculations for the considered ions seem to be very promising to investigate the relativistic dependency in these quantities for the study of possible variation of $\alpha_{e}$ by determining the sensitivity coefficients $q$ of the transitions among the calculated states. These coefficients are given in Table I with the DC Hamiltonian and with other relativistic corrections using the $\operatorname{CCSD}(\mathrm{T})$ method. The obtained results are quite enhanced in these ions and the values increase for the heavier ions. We also observe that the corrections due to the Breit and QED interactions are influencing the results considerably which are never investigated before in the other studied highly charged ions 21, 22]. It can be found that the contributions from the QED corrections are almost negligible in the $\Delta E_{a}$ calculations, however these contributions are found to be relatively large in the determination of $q$ values. Since our calculated $\Delta E_{a}$ values are below $0.5 \%$ accurate compared with their experimental values, on this ground we recommend that these reported $q$ values are also accurate within the same percentage.

We now turn to determining other properties of the transitions whose sensitivity coefficients are estimated in this work. The important transition properties that should be known precisely for their astrophysical observations are the transition probabilities, the oscillator strengths and the lifetimes of the considered excited states. The transitions from the fine structure level to the ground state in these ions decay through the M1 and E2 forbidden channels while the $3 s 3 p^{6}{ }^{2} S_{1 / 2}$ state decay to the $3 s^{2} 3 p^{5}{ }^{2} P_{1 / 2}$ state and to the ground state via the E1 channel. The transition amplitudes for these channels obtained from our calculations with different approximations are given in Table III] At the end, we also give the recommended values as "Reco" with maximum probable uncertainties associated with these values. These uncertainties are estimated based on the intuitive guess from the trends they exhibit using the CCSD and $\operatorname{CCSD}(\mathrm{T})$ methods and at various approximations in the Hamiltonian. It can also be noticed that the DHF results are large from the RCC calculations and the differences between the DHF and RCC results are small for the M1 transition amplitudes which are large in the E2 amplitudes and the RCC results are almost half of the DHF results in the E1 amplitudes persuading large correlation effects in this property. To understand the role of various correlation effects in the RCC calculations of these quantities, we give contributions explicitly from various terms of the $\operatorname{CCSD}(\mathrm{T})$ method using the full Hamiltonian in Tables [V and $\mathrm{V}$. As can be seen, the effective one-body contribution through $\bar{O}$ involving the DHF result is the most dominant contributing term followed by the effective two-body terms for the M1 transition else the $\bar{O}_{o b} R_{2 a}$ term along with its $c c$ term in the E1 and 
E2 amplitude calculations. The reason for $\bar{O}_{o b} R_{2 a}$ and effective two-body contributions being very large as they account directly the core-polarization contributions to all orders involving the valence electrons which are found to be very crucial in the considered ions. Nevertheless, the contributions from norm are non-negligible.

Using the above transition amplitudes, we give the transition probabilities and the oscillator strengths for the considered ions in Table VI. To estimate these quantities, we have used the experimental energies to avoid the uncertainties coming out from the calculated energies although these calculations are sufficiently accurate to provide precise $a b$ initio values. There are estimation of the transition probabilities due to the M1 transitions earlier 48] which were determined using the M1 amplitudes obtained using the DHF method and experimental energies. Since correlation effects are very small in the calculation of the M1 amplitudes in the considered transitions, we see a very good agreement between both the work. Recently, the transition probability of the $3 s^{2} 3 p^{5}{ }^{2} P_{1 / 2} \rightarrow 3 s^{2} 3 p^{5}{ }^{2} P_{3 / 2}$ transition of the Fe $\mathrm{X}$ ion is measured by Brenner et al. [49] which also agree with our result. But our result seem to be more precise than these two reported values. The oscillator strengths for the allowed transitions are found to be large enough to be used for the detection of these lines in the astrophysical observations.

Finally, we present the lifetimes of the excited states in Table VII These values are compared with the previously reported experimental and predicted values. As seen in the table, the experimental values have large uncertainties except for the $3 s^{2} 3 p^{5}{ }^{2} P_{1 / 2}$ state of Ni XII. The lifetime of the $3 s^{2} 3 p^{5}{ }^{2} P_{1 / 2}$ state of Fe $\mathrm{X}$ was measured in a Kingdon ion trap [59, 60] which differs from other measurements that are carried out optically in a heavy-ion storage ring [61] and using the electron beam ion-trap technique [49]. Our result agrees with the latter two measurements. The lifetimes for the same state in Co XI and Ni XII are also measured by Träbert and coworkers 61] which agree with our estimated values, however our theoretical values seem to be more precise than the measurements. The other predicted values of the lifetime of this state in all these three ions $31,50-58$ are either obtained from the astrophysical observations or estimated using lower order many-body methods than our RCC method. We could not find out any reported values for the lifetimes of the above two excited states of Mn IX in the literature. Our estimated results for the lifetimes of the excited states in this ion will be useful for their measurements.

\section{CONCLUSION}

We have developed a relativistic coupled-cluster method to calculate atomic wave functions of the states in ions which have one electron less than the closedshell electronic configurations. We successfully employed this theory to calculate the wave functions in the highly charged Mn IX, Fe X, Co XI and Ni XII ions. The DiracCoulomb Hamiltonian with other relativistic interactions such as Breit, vacuum polarization and self energy corrections is used to incorporate both the relativistic and correlation effects more rigorously in the calculations. Configuration interaction space is approximated at the singles and doubles excitation level, however they are elevated by the inclusion of the important triple excitations in a self-consistent manner through a perturbative approach. We have obtained the detachment energies within sub-one percent accuracy and estimated the sensitivity coefficients for the investigation of any possible temporal variation of the fine structure constant at the same level of accuracy. Roles of various relativistic and correlation effects are demonstrated explicitly. Further more, we determined the transition matrix elements due to the E1, M1 and E2 channels from the considered excited states in the above ions. Using these matrix elements, we evaluated the transition probabilities, the oscillator strengths and the lifetimes of the excited states and compared them with the available experimental and other predicted values. Our estimated results are found to be more precise than the previously reported results. The corresponding experimental results for some of our reported values are not known, hence our calculated values will serve as the benchmark results for their future measurements.

\section{Acknowledgment}

We thank Yashpal Singh for his partial contributions in the developed method. The calculations were carried out using PRL 3TFLOP HPC cluster, Ahmedabad.
[1] J. P. Uzan, Rev. Mod. Phys. 75, 403 (2003)

[2] N. Leefer, C. T. M. Weber, A. Cingöz, J. R. Torgerson and D. Budker, arXiv: 1304.6940.

[3] J. A. King, J. K. Webb, M. T. Murphy, V. V. Flambaum, R. F. Carswell, M. B. Bainbridge, M. R. Wilczynska and F. E. Koch, MNRAS 422, 3370 (2012).

[4] H. Chand, R. Srianand, P. Petitjean and B. Aracil, Precision Spectroscopy in Astrophysics, Proceedings of the
ESO/Lisbon/Aveiro Conference held in Aveiro, Portugal, 1115 September 2006, pp. 101 (2008).

[5] T. Damour, F. Piazza and G. Veneziano, Phys. Rev. D 89, 081601 (2002).

[6] T. Damour, F. Piazza and G. Veneziano, Phys. Rev. D 66, 046007 (2002).

[7] T. Kaluza, Sitzungber. Press. Akad. Wiss. Phys. Math. Kl. L IV, 966 (1921). 
[8] O. Klein, Z. Phys. 37, 895 (1926).

[9] A. Chodos and S. Detweiler, Phys. Rev. D 21, 2167 (1980).

[10] W. J. Marciano, Phys. Rev. Lett. 52, 489 (1984).

[11] K. A. Bronnikov, S. A. Kononogov and V. N. Mel'nikov, Measurement Tech. 56, 8 (2013).

[12] T. Damour and A. M. Polyakov, Nucl. Phys. B 423, 532 (1994).

[13] N. N. Kolachevskil, Phys. Uspekhi 47, 1101 (2004).

[14] D. Prestage, R. L. Toelker and L. Maleki, Phys. Rev. Lett 74, 3511 (1995).

[15] M. J. Drinkwater, J. K. Webb, J. D. Barrow and V. V. Flambaum, MNRAS 295, 457 (1998).

[16] J. K. Webb, V. V. Flambaum, C. W. Churchill, M. Drinkwater and J. D. Barrow, Phys. Rev. Lett. 82, 884 (1999).

[17] L. L. Cowie and A. Songaila, Astrophys. J. 453, 596 (1995).

[18] J. Bahcall, W. L. W. Sargent and M. Schmidt, Astrophys. J. L 11, 149 (1967).

[19] M. T. Murphy, V. V. Flambaum, J. K. Webb, V. V. Dzuba, J. X. Prochaska and A. M. Wolfe, Lect. Notes Phys. Springer-Verlag, Berlin 648, 131 (2004).

[20] S. A. Levshakov, Lect. Notes Phys., Springer-Verlag, Berlin 648, 151 (2004).

[21] J. C. Berengut, V.A. Dzuba, V.V. Flambaum and A. Ong, Phys. Rev. Lett. 109, 070802 (2012).

[22] J. C. Berengut, V.A. Dzuba and V.V. Flambaum, Phys. Rev. Lett. 105, 120801 (2010).

[23] http://physics.nist.gov/cgi-bin/ASD/energy1.pl

[24] B. Edlen, Z. Astrophy. 22, 30 (1942).

[25] K. P. Dere, Astrophys. J. 221, 1062 (1978).

[26] R. J. Thomas and W. M. Neupert, Astrophys. J. Suppl. 91, 461 (1994).

[27] H. Nussbaumer, Astron. Astrophys. 48, 93 (1976).

[28] A. K. Bhatia and G. A. Doschek, At. Data Nucl. Data Tables 60, 97 (1995).

[29] G. Del Zanna, K. A. Berrington and H. E. Mason, Astron. Astrophys. 422, 731 (2004).

[30] C. Jordan, Phys. Lett. 18, 259 (1965).

[31] H. E. Mason and H. Nussbaumer, Astron. Astrophys. 546547 (1977).

[32] B. C. Fawcett and A. T. Hatter, Astron. Astrophys. 84, 78 (1980).

[33] B. C. Fawcett and R. W. Hayes, J. Phys. B: Atomic, Mol. and Optical Phys. 5, 366 (1972).

[34] B. C. Fawcett, R. D. Cowan and R. W. Hayes, J. Phys. B: At. Mol. Opt. Phys. 5, 2143 (1972).

[35] http://www.pa.uky.edu/ peter/atomic

[36] A. J. J. Raassen, R. Mewe, M. Audard, M. Güdel, E.
Behar, J. S. Kaastra, R. L. J. van der Meer, C. R. Foley and J. -U. Ness, Astron. Astrophys. 389, 228 (2002).

[37] W. Greiner, Relativistic Quantum Mechanics, 3rd edition, Springer-Verlag, Berlin, Germany (2000).

[38] V. A. Dzuba, V. V. Flambaum and J. K. Webb, Phys. Rev. A 59, 1 (1998).

[39] W. R. Johnson, Atomic Structure Theory, SpringerVerlag (Berlin) (2007).

[40] I. I. Sobelman, Atomic Spectra and Radiative Transitions, Springer-Verlag (Berlin), pp. 223 (1979).

[41] G. Breit, Phys. Rev. 39, 616 (1932).

[42] V. V. Flambaum and J. S. M. Ginges, Phys. Rev. A 72, 052115 (2005)

[43] V. M. Shabaev, Phys. Rev. A 64, 052104 (2001).

[44] I. Shavitt and R. J. Bartlett, Many-body methods in Chemistry and Physics, Cambidge University Press, Cambridge, UK (2009).

[45] B. K. Sahoo, S. Majumder, R. K. Chaudhuri, B. P. Das and D. Mukherjee, J. Phys. B: At. Mol. Opt. Phys. 37, 3409 (2004).

[46] D. Mukherjee, B. K. Sahoo, H. S. Nataraj and B. P. Das, J. Phys. Chem. A 113, 12549 (2009).

[47] I. Lindgren and J. Morrison, Atomic Many-body Theory, second edition, Springer-Verlag, Berlin, Germany (1986).

[48] V. Kaufman and J. Sugar, J. Phys. Chem. Data 15, 321 (1986).

[49] G. Brenner et al., Astrophys. J. 703, 68 (2009).

[50] T. K. Krueger and S. J. Czyzak, Astrophys. J. 144, 1194 (1966).

[51] B. Warner, Z. Astrophys. 69, 399 (1968).

[52] M. W. Smith and W. L. Wiese, J. Phys. Chem. Ref. Data 2, 85 (1973).

[53] S. O. Kastner, Sol. Phys. 46, 179 (1976).

[54] M. Kafatos, and J. P. Lynch, Astrophys. J. Suppl. 42, 611 (1980).

[55] M. Eidelsberg, F. Crifo-Magnant and C. J. Zeippen, Astron. Astrophys. Suppl. 43, 455 (1981).

[56] K-N. Huang, Y-K. Kim, K. T. Cheng and J. P. Desclaux, At. Data Nucl. Data Tables 28, 355 (1983).

[57] C. Kohstall, S. Fritzsche, B. Fricke, W-D. Sepp and E. Trabert, Phys. Scr. T. 80, 482 (1999).

[58] C. Dong, S. Fritzsche, B. Fricke and W-D. Sepp, Mon. Not. R. Astron. Soc. 307, 809 (1999).

[59] D. P. Moehs, M. I. Bhatti and W. F. Perger, Phys. Rev. Lett. 84, 38 (2000).

[60] D. P. Moehs, M. I. Bhatti and D. A. Church, Phys. Rev. A 63, 032515 (2001).

[61] E. Träbert, G. Saathoff and A. Wolf, J. Phys. B: At. Mol. Opt. Phys. 37, 945 (2004). 